\title{
Persimmon (Diospyros Kaki Thunb 'Saijo') Peel Improved Dyslipidemia and its Related Production of Atherogenic Autoantigen Complexes in Low- Density Lipoprotein Receptor-Deficient Mice
}

\author{
Nanhu Quan ${ }^{\#, 1,2}$, Kazuko Kobayashi ${ }^{\#, 2}$, Yukana Matsunami ${ }^{2}$, Masahiro Ide ${ }^{2}$, Marina Makarova ${ }^{2,3}$, \\ Lianhua Shen ${ }^{2,4}$, Shoichi Ohno ${ }^{5}$, Yang Zheng ${ }^{1}$, Haruo Kobayashi ${ }^{6}$, Luis R. Lopez ${ }^{7}$ and \\ Eiji Matsuura*,2 $^{*}$
}

${ }^{1}$ Cardiovascular center, First Hospital, Jilin University, Changchun 130021, China; ${ }^{2}$ Departments of Cell Chemistry; ${ }^{3}$ Department of Pathology, I.M. Sechenov Moscow Medical Academy, Moscow, Russia; ${ }^{4}$ Bacteriology, Okayama University Graduate School of Medicine, Dentistry, and Pharmaceutical Sciences, 2-5-1 Shikata-cho, Kita-ku, Okayama 700-8558, Japan; ${ }^{5}$ Okayama Agriculture Development Institute, Akaiwa, 709-0801, Okayama; ${ }^{6}$ Regional Cooperative Research Organization, Okayama Prefectural University, Soja, 719-1197, Okayama; ${ }^{7}$ Corgenix Inc, Bromfield, CO, USA

\begin{abstract}
Role of persimmon (Diospyros kaki, Thunb 'Saijo') peel was investigated on developing atherosclerosis in low-density lipoprotein receptor (LDLR)-deficient mice with emphasis on lipid metabolism, physio-biological oxidation, production of related atherogenic autoantigens, and production of anti-atherogenic natural antibodies. Male LDLRdeficient mice were fed high fat diet or high fat diet supplemented with $10 \%$ dried and powdered persimmon peel (PP) for 12 weeks. PP supplementation significantly prevented the increment of plasma cholesterol and triglyceride levels. High fat diet feeding increased plasma level of oxidized LDL/ $\beta 2$-glycoprotein I (oxLDL/ $\beta 2 \mathrm{GPI}$ ) complexes regarded as an atherogenic autoantigen, while PP supplementation significantly blocked the increment $(p<0.05)$. After a 12 -week feeding, atherosclerotic plaques in mice fed with diet supplemented with PP decreased by $70 \%$ as compared to that of mice fed the high fat diet $(p<0.005)$. PP feeding also reduced urinary 11-dehydro-thromboxane $\mathrm{B}_{2}$, a stable metabolite of the platelet activation marker thromboxane $\mathrm{A}_{2}$, but level of IgM anti-oxLDL antibodies was not changed. Thus, these results obviously demonstrate that persimmon peel may have an anti-atherogenic property through normalization of lipid metabolism and reduced production of the atherogenic complexes.
\end{abstract}

Keywords: Persimmon peel, oxidized low-density, lipoprotein, $\beta 2$-glycoprotein I, atherosclerosis, II-dehydro-thromboxane $\mathrm{B}_{2}$.

\section{INTRODUCTION}

The fruit persimmon (Diospryros kaki) contains several bioactive compounds, such as polyphenols, flavonoids, terpenoids, steroids, dietary fiber, carotenoids and minerals [1]. Though persimmon peel (PP) is rich in carotenoids and polyphenols rather than persimmon pulp [2], the peel is discarded during production of dried fruit. It has been reported that PP components have beneficial effects such as antioxidant activity [3-5], and tyrosinase inhibiting activity (whitening activity) [6]. It has also been reported that young persimmon fruit has hypolipidemic effect $[7,8]$. In the present study we examined the effect of PP on dyslipidemia, atherogenesis, and production of immune-regulated components in hypercholesterolemic mouse model to evaluate the possibility that PP is a food supplement against atherogenesis.

Atherosclerosis is a chronic inflammatory disease that results from disturbed lipoprotein metabolism, the formation of pro-inflammatory lipid peroxidation products, and host's

*Address correspondence to this author at the Department of Cell Chemistry, Okayama University Graduate School of Medicine, Dentistry, and Pharmaceutical Sciences, Japan; Tel: +81-86-235-7402; Fax: +81-86-2357404; E-mail: eijimatu@md.okayama-u.ac.jp

\#These authors are contributed equally to this work. immune responses. In vitro observations suggest that native low-density lipoprotein (LDL) itself does not induce any features associated with atherosclerosis (e.g. formation of lipid-laden form cells from macrophages) but oxidatively modified LDL (oxLDL) and its byproducts are highly proinflammatory and pro-atherogenic $[9,10]$.

OxLDL accumulation in macrophage-derived foam cells in atherosclerotic lesions has been detected using antioxLDL antibodies $[11,12]$. OxLDL in human circulation has also been detected using anti-oxLDL antibodies and antiapolipoprotein B (apoB) antibodies [13-15]. OxLDL contains a wide variety of oxidation-specific epitopes that makes it an excellent immunogen. These oxidation specific epitopes lead to profound immune responses including autoantibody production that modulate lesion formation. Natural antibodies, mainly IgM responses to oxLDL have been found and some of them cloned [16-18]. Some autoantibodies to oxLDL derived from 'naive' atherosclerotic mice share complete genetic and structural identity with antibodies from the classic anti-phosphorylcholine B-cell clone T15, which protects against common infectious pathogens including pneumococci [19].

We demonstrated that oxLDL/ $\beta 2 \mathrm{GPI}$ complex is a major atherogenic and thrombogenic autoantigen in patients with 
antiphospholipid syndrome (APS), a systemic autoimmune disease [20-24], and that elevated level of oxLDL/B2GPI complexes was detected in patients with systemic autoimmune diseases, diabetes mellitus, and chronic renal disease [25-28]. OxLDL/32GPI induced $\beta 2$ GPI specific autoreactive T cells while soluble $\beta 2$ GPI alone did not $[29,30]$.

Mice lacking LDL receptor (LDLR) have less overt disease than apolipoprotein E (ApoE)-deficient mice [31]. Very low-density lipoprotein (VLDL) formed in the liver is partially metabolized by lipoprotein lipase, generating plasma intermediate density lipoprotein (IDL) particles. Usually, IDL is cleared by the liver via LDLR. When LDLR is defective, IDL remains in the circulation where they are converted to LDL. LDLR-deficient mice have a modest 2 fold-higher plasma cholesterol level (than normal C57BL/6 mice) when maintained on a normal chow diet, and they only develop atherosclerotic plaques slowly [32]. However, in response to a high-fat, high-cholesterol diet, LDLR-deficient mice exhibit massive elevations in plasma cholesterol and rapidly develop atherosclerotic lesions throughout the aorta. In contrast, ApoE deficient mice develop lesions while being fed a chow diet. We considered it would be convenient to use LDLR-deficient mice to examine the dietary effect, as most part of their lesions would be generated by the effect of diet.

In this study, we measured the level of oxLDL/ $22 \mathrm{GPI}$ complexes in LDLR-deficient mice fed a high fat diet and the PP-supplemented high fat diet. The objectives of the current study were to determine whether a diet containing PP could alter the progression of atherosclerosis in the LDLRdeficient mice, influence blood lipid levels, markers of oxidative stress such as $8-\mathrm{OHdG}$, atherogenic autoantigen complexes, and natural antibody production against oxLDL.

\section{MATERIALS AND METHODS}

\subsection{Diets}

In this study, we used three types of diet. The first was a normal chow diet (MF, Oriental Yeast, Co. Ltd. Tokyo, Japan) containing $0.08 \%$ cholesterol and a high fat diet. The second was a high fat diet (HF) containing $0.2 \%$ cholesterol and $21 \%$ milk fat (Oriental Yeast). The third diet was HF containing $10 \%$ dried persimmon (Diospyros Kaki Thunb. 'Saijo') peel (PP) powder, which was designated as HF + PP. $\mathrm{PP}$ was sun-dried for 30 days and further dried with vacuum dryer (BCD-2000U; Yahiro Industries, Minokamo, Japan) at $40^{\circ} \mathrm{C}$ for $14 \mathrm{~h}$, followed by milling with pinmill (SogoSangyo, Tokyo, Japan). The resulting powder was added at $10 \%$ in HF. The macronutrient composition of the dried persimmon peel powder and experimental diets are shown in Table 1. Total calories were calculated according to Atwater energy equivalent.

\subsection{Animals and Experimental Protocol}

LDLR deficient mice (B6, 129S7-Ldlr $\left.{ }^{\mathrm{tm} 1 \mathrm{Her}} / \mathrm{J}\right)$ were purchased from Jackson Laboratory (Bar Harbor, ME, USA). Three to five animals were housed per cage in a temperaturecontrolled animal facility with a daily photoperiod of $12 \mathrm{~h}$ of light at the Department of Animal Resources, Advanced Science Research Center, Okayama University. Mice fed normal chow diet (MF) until the experiment started. At 12 weeks of age, male LDLR deficient mice were randomly assigned to three groups and fed HF $(n=11), H F+P P(n=7)$, or normal chow $(n=10)$ diet for 12 weeks. Besides, eight 12 weeks old male LDLR deficient mice were fed on HF and received acetylsalicyclic acid (aspirin) $(30 \mathrm{mg} / \mathrm{L})$ in their drinking water, which was replaced with fresh water every other day, for 12 weeks. Considering that each animal drinks in average 3 to $4 \mathrm{~mL}$ of water per day, this would be equal to 90 to $120 \mu \mathrm{g}$ aspirin per day for a mouse of $30 \mathrm{~g}$ of weight. On a body scale-adjusted scale, this amount would be equal to 180 to $240 \mathrm{mg} /$ day if the animals weighed $60 \mathrm{~kg}$ [33]. Body weight was measured at the beginning of the study and following every 4 weeks. On the same day for body weight measurement, each mouse was set in a metabolism cage with water but without any foods for 5-6 h. Then urine sample dropped into the container at the bottom was gathered. Right after the urine collection, blood samples were obtained from animals by retro-orbital bleeding with EDTA as an anticoagulant. All animal experiments were performed according to the guidelines of Okayama University and the study protocol was approved by the Committee on Animal Experimentation of Okayama University.

\subsection{Plasma Lipids}

Plasma cholesterol and triglyceride were determined enzymatically using commercial kits (Cholesterol E-test and Triglyceride E-Test, respectively; Wako Pure Chemical Industries, Ltd., Osaka, Japan).

Table 1. Nutritional Data for the Persimmon Peel and Experimental Diets

\begin{tabular}{|c|c|c|c|c|}
\hline Constituents & ND & HF & PP & $\mathbf{H F}+\mathbf{P P}$ \\
\hline Protein $(\%)$ & 27.8 & 17.9 & 4.1 & 16.5 \\
\hline Fat (\%) & 4.9 & 21.0 & 1.4 & 19.0 \\
\hline Carbohydrates (\%) & 48.4 & 49.1 & 71.3 & 51.3 \\
\hline Fiber (\%) & 4.4 & 3.0 & 12.7 & 4.0 \\
\hline Ash (\%) & 7.7 & 2.0 & 2.5 & 2.1 \\
\hline Moisture (\%) & 6.8 & 7.0 & 8.0 & 7.1 \\
\hline Energy (kcal/100g) & 349 & 457 & 314 & 442 \\
\hline
\end{tabular}

ND, Normal mouse chow diet (MF, Oriental Yeast); HF, a high fat diet containing $21 \%$ milk fat, $0.2 \%$ cholesterol; PP, dried and powdered persimmon peel; HF + PP, HF added with $10 \% \mathrm{PP}$. 


\subsection{Preparation of Mouse Aortas and Quantification of Atherosclerosis}

Mice were euthanized after the final blood collection at 24 weeks of age. The aortic tree was perfused with phosphate buffered saline (PBS) containing $20 \mu \mathrm{M} / \mathrm{L}$ butylated hydroxytoluene (BHT) and $2 \mu \mathrm{M}$ EDTA, $\mathrm{pH} 7.4$, by inserting a cannula into the left ventricle and allowing free efflux from an incision in the vena cava. After removal of the surrounding adventitial fat tissue, the aorta was opened longitudinally from the aortic root to the iliac bifurcation, fixed with PBS containing $4 \%$ formaldehyde, and stained with Sudan IV. The extent of atherosclerosis was determined by the "en face" method [34]. Quantification was performed by capturing the images of aortas with Penguin 150 CL camera (Pixera Corp. San Jose, CA) connected to SZX12 dissection microscope (Olympus Corp. Tokyo, Japan). The lesion percent of aorta was estimated by Scion Image analysis.

\subsection{Preparation and Oxidation of Mouse LDL}

LDL fraction was collected from another group of LDLR-deficient mice to prepare oxLDL/32GPI complexes for an ELISA. Twelve LDLR-deficient mice fed HF for more than 4 weeks were anesthetized with ethyl ether and blood was drawn from the right ventricle using EDTA as an anticoagulant. The plasma was separated by centrifugation and pooled. LDL was separated by sequential ultracentrifugation at 550,000 $\mathrm{x}$ g adjusting the density with potassium bromide as described previously [13], using TL-100 ultracentrifuge (Beckman Coulter) and TLA100.3 rotor. After LDL fraction was dialyzed against PBS to remove EDTA and potassium bromide, it was catalytically oxidized with 5 $\mu \mathrm{M} \mathrm{CuSO}{ }_{4}$ for 16 hours at $37^{\circ} \mathrm{C}$ as described before [24]. The oxidation was terminated by adding EDTA at final concentration of $1 \mathrm{mM}$. Aliquots were taken and subjected to assay for thiobarbituric acid-reactive substance (TBARS) and agarose gel electrophoresis. OxLDL/32GPI complex as a calibrator was prepared by incubating mouse oxLDL and human $\beta 2 \mathrm{GPI}$ at the ratio of $2: 1(\mathrm{w} / \mathrm{w})$ for $12 \mathrm{~h}$ at $37^{\circ} \mathrm{C}$. The oxLDL and oxLDL/ $32 \mathrm{GPI}$ complexes were stored at $-80^{\circ} \mathrm{C}$ until use.

\subsection{Antibodies}

Mouse anti- $\beta 2$ GPI monoclonal antibody WB-CAL- 1 was prepared as described previously [35]. Rabbit anti-mouse LDL polyclonal antibodies were prepared as follows. Briefly, Japanese white rabbits were immunized with the LDL obtained from LDLR-deficient mice together with Freund's Incomplete Adjuvant (Sigma-Aldrich, St. Louis, MO, USA) and boosted four times following every two weeks. The antibody titer of rabbit sera was checked with an ELISA against mouse native LDL and mouse oxLDL. IgG of mouse LDL antibody was purified by protein A-Sepharose CL-4B (GE Healthcare UK Ltd. Buckinghamshire, UK), and labeled with horseradish peroxidase (HRP) (Seikagaku Co., Tokyo, Japan).

\subsection{ELISA for Mouse oxLDL/ $\beta 2$ GPI Complexes}

The ELISA for mouse plasma oxLDL/ $\beta 2$ GPI complex was performed as follows, with slight modification of human assay system [25]. Anti- $\beta 2$ GPI monoclonal antibody WBCAL-1 $(8 \mu \mathrm{g} / \mathrm{ml})$ was adsorbed on the wells of 96-well microtiter plate (Maxisorp, Nunc) by overnight incubation at $4^{\circ} \mathrm{C}$. Then the wells were blocked with BSA for $2 \mathrm{~h}$. Mouse plasma (100-fold diluted) or oxLDL/ $\beta 2$ GPI complexes as a calibrator (8-800 $\mathrm{ng} / \mathrm{ml}$ apoB equivalent) were incubated at $4^{\circ} \mathrm{C}$ overnight. The wells were subsequently incubated with HRP-labeled rabbit anti-mouse LDL antibodies for $3 \mathrm{~h}$ at room temperature. Color was developed with adding TMBUS substrate (Moss Inc, Pasadena MD, USA). The reaction was terminated by adding $2 \mathrm{~N}$ sulfuric acid and the $\mathrm{OD}$ at $450 \mathrm{~nm}$ was measured. Between each step, the wells were extensively washed with TBS containing $0.05 \%$ Tween 20. The intra-assay precision (CV) was less than $10 \%$.

\subsection{ELISA for 11-Dehydrothromboxane $B_{2}$ (11-dhTXB $\left.{ }_{2}\right)$}

Urinary 11-dhTXB ${ }_{2}$ was determined using $11-\mathrm{dhTXB}_{2}$ test kit (Corgenix, Broomfield CO, USA). The 10-fold diluted urine samples, 11-dhTXB 2 conjugated to alkaline phosphatase (AP), and purified mouse monoclonal antibody directed to $11-\mathrm{dhTXB}_{2}$ were incubated together in wells coated with a polyclonal anti-mouse antibody. Incubation allowed the endogenous 11-dhTXB 2 present in the samples to compete with the purified AP-conjugated 11-dhTXB 2 to the bound anti-11-dhTXB ${ }_{2}$ antibody. After washing, paranitrophenylphosphate (pNPP) chromogenic substrate was added and color developed in the wells at an intensity inversely proportional to the urine sample concentration of 11$\mathrm{dhTXB}_{2}$. The concentration of 11-dhTXB 2 was adjusted by urinaly creatinine. Creatinine concentration was determined with Creatinine Assay Kit (Cayman Chemical Company Ann Arbor, MI, USA).

\subsection{8-Hydroxy-2'-Deoxyguanosine (8-OHdG) and Adi- ponectin Measurement}

Urinary 8-OHdG levels were determined using an ELISA kit according to the manufacturer's instructions (Japan Institute for the Control of Aging, Fukuroi, Japan). Mouse plasma adiponectin levels were measured using an ELISA kit according to the manufacturer's instructions (Otsuka Pharmaceutical Co Ltd, Tokyo, Japan).

\subsection{ELISA for IgM Anti-oxLDL Antibody}

The ELISA to measure mouse IgM anti-oxLDL was performed as follows. Mouse oxLDL $(2 \mu \mathrm{g} / \mathrm{ml})$ was adsorbed on the wells of a 96-well microtiter plate (Immulon 1B, Dynex Technologies, Chantilly, VA, USA) by overnight incubation at $4^{\circ} \mathrm{C}$. Then the wells were blocked with BSA for $2 \mathrm{~h}$. Mouse plasma (100-fold diluted) was added and incubated at $37^{\circ} \mathrm{C}$ for $1 \mathrm{~h}$ at room temperature. The wells were subsequently incubated with HRP-labeled goat anti-mouse $\operatorname{IgM}$ (American Qualex, San Clemente, CA, USA) for $1 \mathrm{~h}$ at room temperature. Further steps were performed in the same way as the ELISA for oxLDL/ $\beta 2$ GPI complexes.

\subsection{Statistical Analysis}

Data are presented as means + /- SD. One way analysis of variance (ANOVA) and Dunnet analysis as post hoc were used to determine significant differences at $p<0.05$ among 3 groups or more with KaleidaGraph software Version 4.0 (Synergy Software, Reading, PA, USA). Unpaired $t$ test was used to determine significant differences at $p<0.05$ between two groups. 


\section{RESULTS}

\subsection{Body Weight and Blood Lipids}

Changes in the body weight of LDLR-deficient mice are shown in Fig. (1A). In HF and HF + PP groups, body weight significantly increased compared to that of the MF group $(p<0.05$, at 24 weeks of age). There was no significant difference in either the initial or the final body weight between $\mathrm{HF}$ and HF + PP groups. After 4 weeks and 8 weeks of feeding, plasma cholesterol level in the HF + PP group was significantly lower than that in the HF group (Fig. 1B, $p<0.005)$. Plasma triglyceride level of HF + PP group was significantly lower than that in the HF group at 20 weeks of age $(p<0.005)$.

\subsection{Plasma oxLDL/ß2GPI Level}

Plasma oxLDL/B2GPI complex levels in LDLR-deficient mice fed HF and HF + PP significantly increased after high fat loading, but the complexes in LDLR-mice fed MF stayed in the level similar to the beginning of the experiment (Fig. 1D). HF feeding led to significant increment in plasma oxLDL/32GPI complexes $(p<0.0005)$, while HF + PP feeding resulted in significant decrease $(p<0.05)$ at 24 weeks of age.

\subsection{Atherosclerotic Lesions}

Mice were euthanized at the end of the experiment. And their aortas were analyzed for the extent of atherosclerosis. Atherosclerotic lesions in the entire arterial tree and in each section of aorta were quantified by the en face method with Sudan IV stain. Moderate atherosclerosis lesions were observed in LDLR-deficient mice fed HF. PP supplementation reduced the lipid-deposit lesion areas in the entire aortic tree by $70 \%$ compared with mice fed HF (Fig. 2B). Atherosclerotic lesions were also significantly smaller in the aortic arch and abdominal aorta than those in the HF group (Fig. 2C-E).
(A)

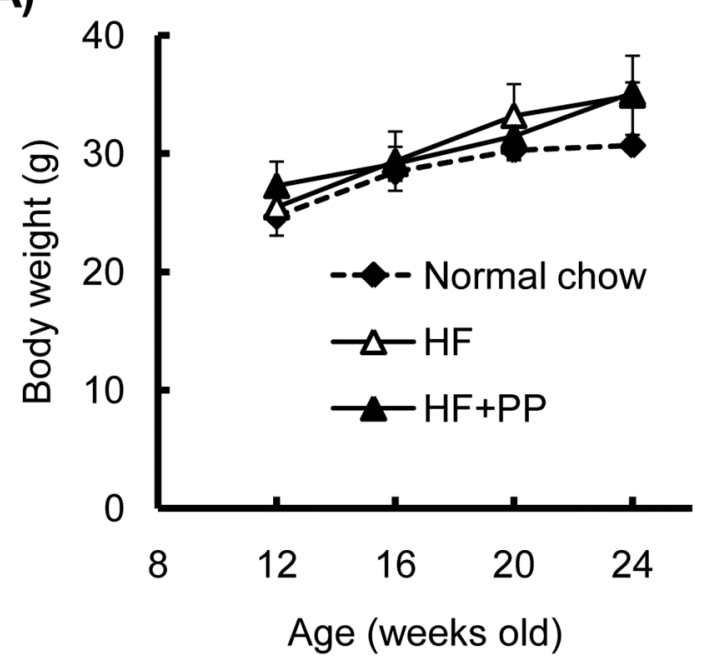

(C)

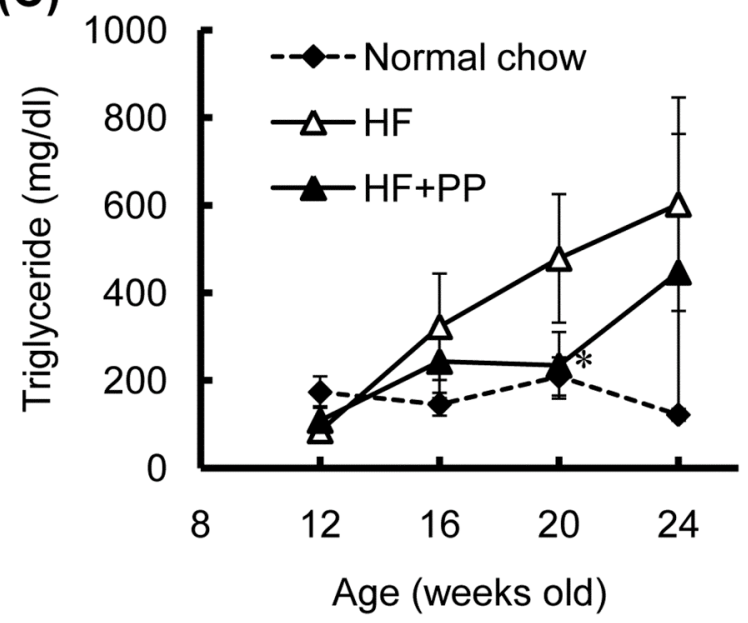

(B)

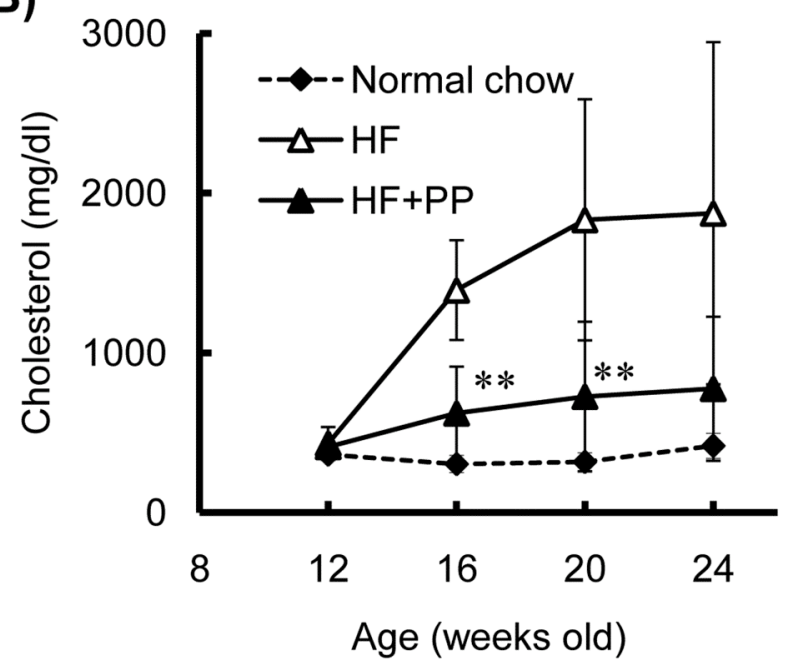

(D)

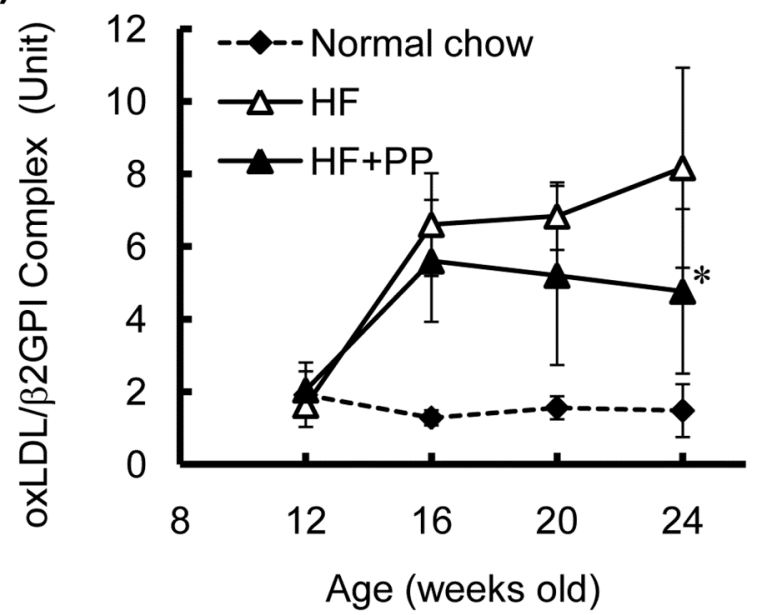

Fig. (1). Change in body weight and plasma parameters of LDLR-deficient mice.

(A) The body weight of LDLR-deficient mice fed HF, HF + persimmon peel (PP), and normal chow diet during the study. (B) Plasma cholesterol level of mice fed HF, HF+PP, and normal chow diet during the study. (C) Plasma triglyceride level of mice fed HF, HF+PP, and normal chow diet during the study. (D) Plasma oxLDL/ $32 \mathrm{GPI}$ complex levels of mice fed HF, HF+PP, and normal chow during the study. Statistical analysis was performed between HF group and HF + PP group of the same age. ${ }^{*} p<0.05$, $* * p<0.005$. Four to eleven mice were applied for each group. 
(A)

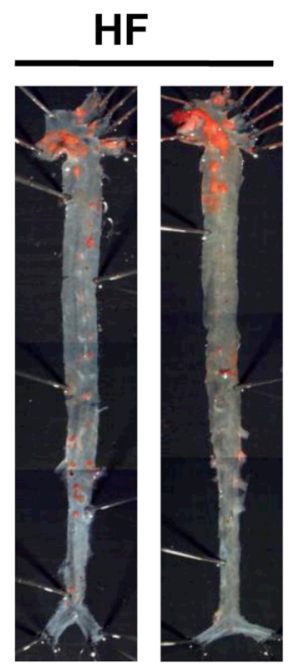

\section{(B) Aortic tree}

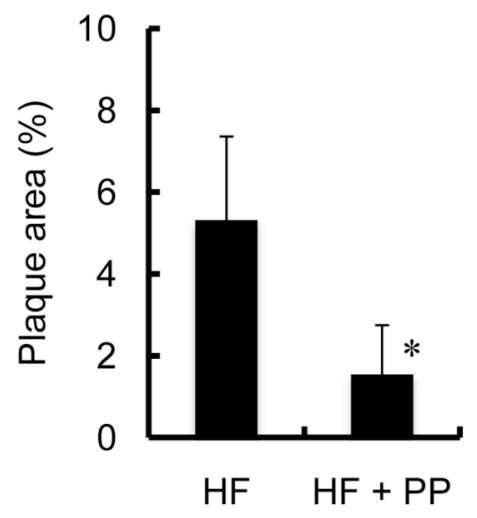

(D) Thoracic aorta

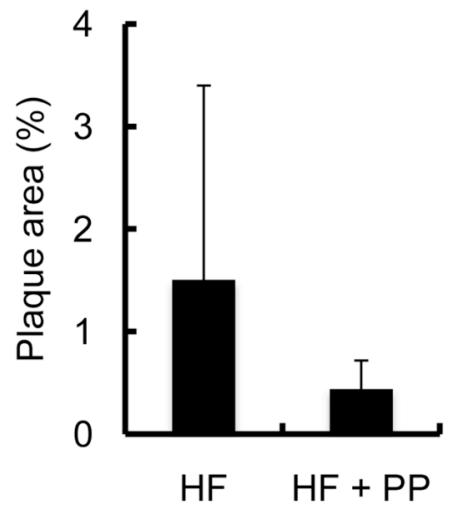

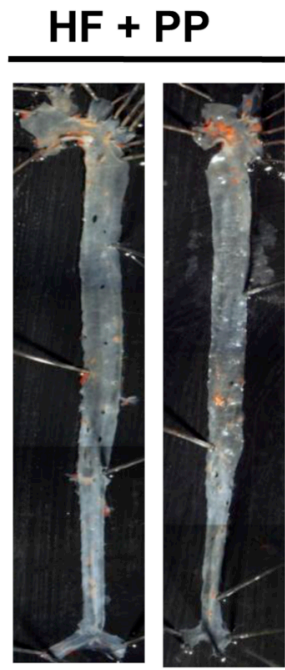

(C) Aortic arch

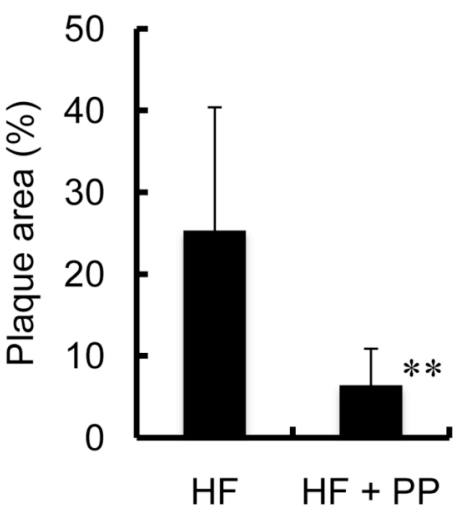

(E) Abdominal aorta

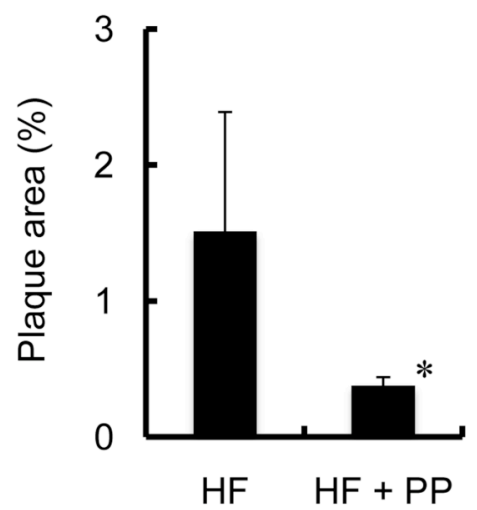

Fig. (2). Atherosclerotic plaque formation in LDLR-deficient mice. (A) Representative atherosclerotic plaques in aortic trees stained with Sudan IV of LDLR-deficient mice fed HF and HF+ persimmon peel (PP). Atherosclerotic lesion size was quantified en face as described in materials and methods and indicated as plaque area in percent of total vessel area (B), aortic arch (C), thoracic aorta (D), and abdominal aorta (E). Statistical analysis was performed between HF group and HF + PP group of the same weeks of age. ${ }^{*} p<0.05, * * p<0.005$

\subsection{Urinary 8-OHdG and Plasma Adiponectin}

Urinary 8-OHdG and plasma adiponectin were measured in LDLR-deficient mice fed HF and HF + PP at 12 weeks of age (baseline) and after 12 weeks of treatment ( 24 weeks of age), respectively (Fig. 3). There was no significant change of urinary 8-OHdG levels and plasma adiponectin levels between control and persimmon supplementation in the beginning, and those between baseline and post-treatment. 
(A)

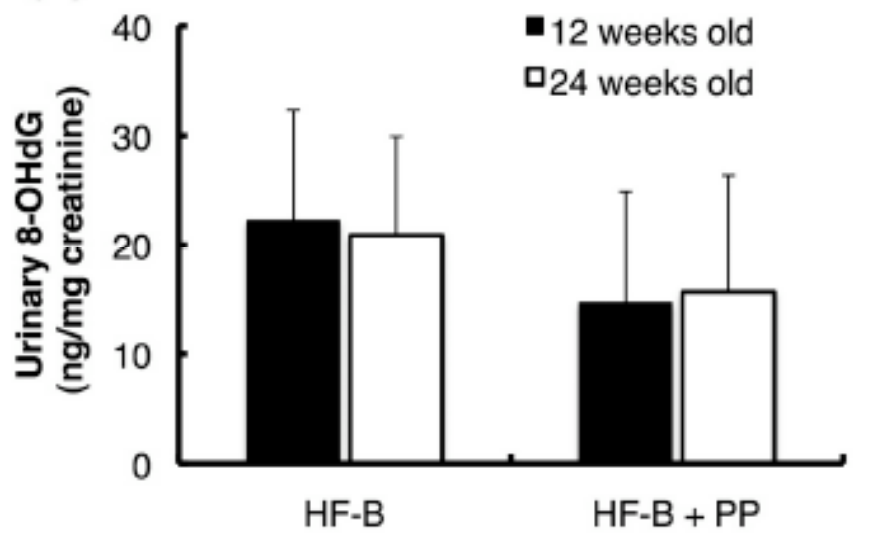

(B)

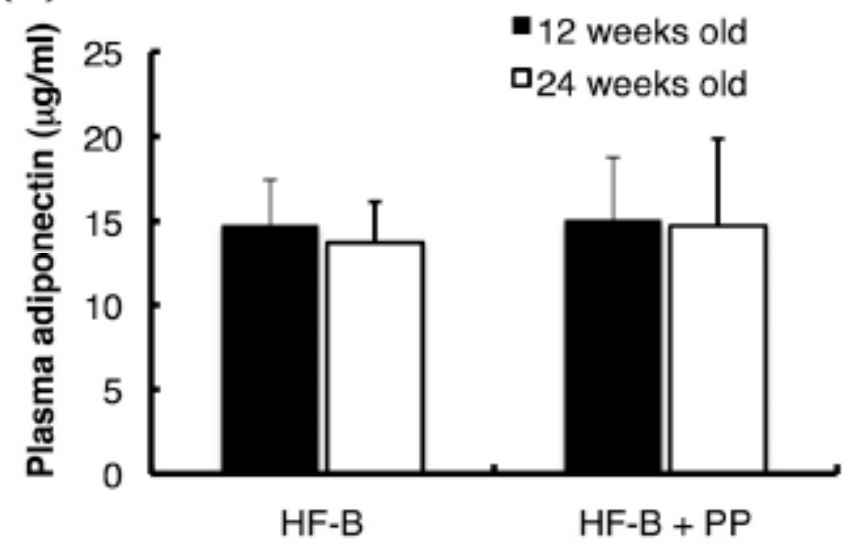

Fig. (3). Oxidation marker and plasma adiponectin in LDLR-deficient mice. (A) Urinary 8-OHdG level in LDLR-deficient mice fed HF and $\mathrm{HF}+$ persimmon peel (PP) at 12 and 24 weeks of age. (B) Plasma adiponectin level in mice fed HF and HF + PP at 12 and 24 weeks of age.

\subsection{Urinary 11-Dehydrothromboxane $B_{2}$}

Mice fed HF showed significant increase in urinary 11dhTXB $_{2}$ (Fig. 4) at 20 weeks of age. Mice fed HF and took aspirin containing water ad libitum (which approximately corresponds to $5 \mathrm{mg} / \mathrm{kg}$ /day) kept the low level of urinary 11-dhTXB 2 throughout the experimental period. After 8 weeks of feeding, persimmon peel supplementation showed significant inhibition of $11-\mathrm{dhTXB}_{2}(p<0.05$, Fig. 4) when compared with HF.

\subsection{Antibody Against oxLDL}

We analyzed IgM antibodies against oxLDL in the plasma of LDLR-deficient mice. The level of antibodies against oxLDL significantly increased over time in mice fed normal chow diet. At 24 weeks of age, antibody levels in mice fed HF were significantly higher than those in mice fed normal chow diet. In LDLR-deficient mice fed HF + PP, the level of antibodies against oxLDL at 20 weeks of age was lower than that in HF, but it was not significant. At 24 weeks of age, antibody levels in HF+ PP largely varied and difference between HF and HF + PP resulted in no statistical significance.

\section{DISCUSSION}

In the present study, we showed that persimmon peel supplementation inhibited the expected increment of plasma cholesterol, triglyceride, and oxLDL/32GPI complexes and decreased the area of atherosclerotic lesions in LDLRdeficient mice. To the best of our knowledge, this is the first report showing significant improvement of atherosclerotic lesion with dietary persimmon peel.

Persimmon peel supplementation prevented the increment of plasma cholesterol and triglyceride. It is necessary to elucidate the mechanism and functional component of lipid-

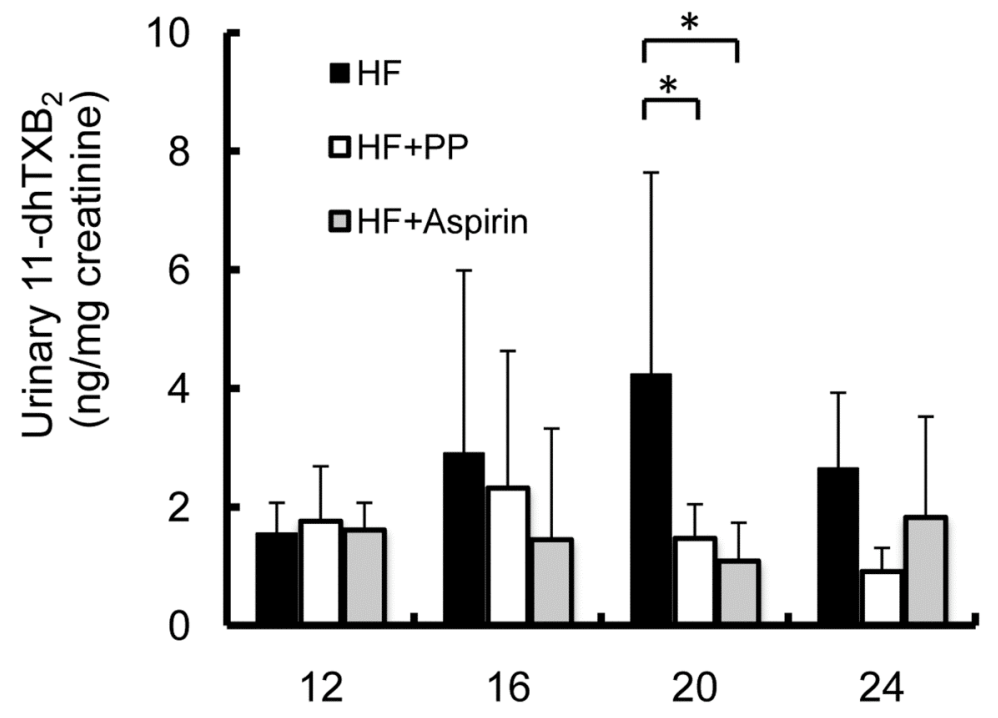

Fig. (4). Urinary 11-dhTXB $\mathrm{devels}_{2}$ in LDLR-deficient mice.

LDLR-deficient mice were fed HF or HF+ persimmon peel (PP) from 12 weeks of age to 24 weeks of age. Mice in HF + Asp group were fed $\mathrm{HF}$ and received aspirin from 12 weeks of age to 24 weeks of age. Urine samples were collected at designated weeks of age and stored at $-80^{\circ} \mathrm{C}$ until use. Urinary $11-\mathrm{dhTXB}_{2}$ level was assayed by ELISA as described in Materials and Methods. ${ }^{*} p<0.05$ compared to HF diet group. 


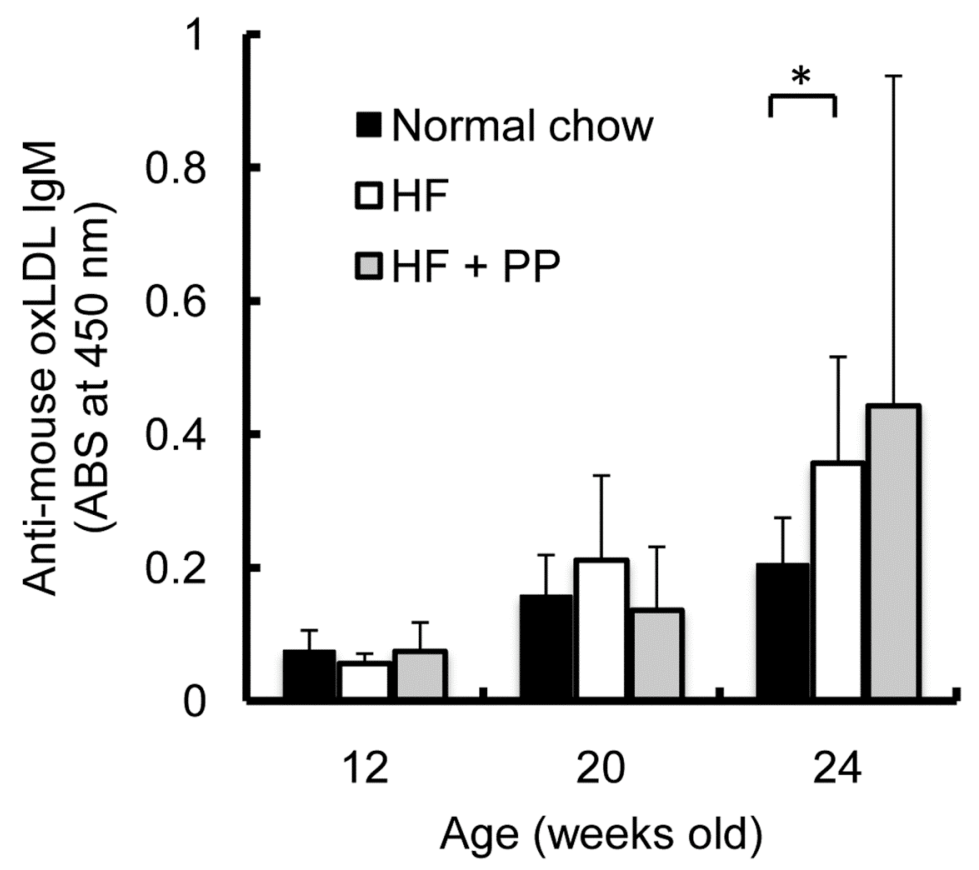

Fig. (5). Plasma anti-mouse oxLDL level in LDLR-deficient mice. IgM anti-oxLDL antibody titer was determined by an ELISA as described in material and methods. ${ }^{*} p<0.05$ compared to normal chow diet group.

lowering effect of persimmon peel. However, some reports about the lipid-lowering effect of persimmon fruits may suggest the mechanism $[7,36]$. The intake of young persimmon fruit reduced the cholesterol level and accelerated fecal bile acid excretion in ApoE-deficient mice [8] and with C57BL/6.Cr mice [37]. They observed increased expression of the hepatic gene for cholesterol $7 \alpha$-hydroxylase (CYP7A1), the rate-limiting enzyme in bile acid biosynthesis. Besides, young persimmon had higher bile-acid binding ability than grown persimmon fruit. The excretion of bile acid into feces is one of the major pathways for the removal of body cholesterol. Fecal bile acids increased significantly with young persimmon treatment, suggesting that enhanced excretion of fecal bile acid prevented hepatic steatosis and the rise in plasma cholesterol levels.

Unlike native LDL, oxLDL binds to $\beta 2 \mathrm{GPI}$ to form immunogenic and pro-atherogenic oxLDL/ $\beta 2$ GPI complexes. We consider that the interaction of oxLDL with $\beta 2 \mathrm{GPI}$ is an anti-oxidant mechanism aimed at neutralizing the effect of oxLDL. OxLDL/B2GPI complexes and autoantibodies to these complexes have been demonstrated in patients with systemic autoimmune disease such as SLE and APS, as well as in patients with non-autoimmune chronic inflammatory conditions such as diabetes mellitus, renal diseases and acute coronary syndrome. In this study, we observed a significant increase in plasma oxLDL/ 32 GPI complexes in LDLRdeficient mice fed HF compared to those fed normal chow diet, at 4 weeks of feeding. This elevation persisted through to the end of the experiment. Persimmon peel supplementation significantly lowered the complex level compared to HF $(\mathrm{p}<0.05)$. Kato et al. [38] reported that plasma oxLDL in ApoE-deficient mice fed on a chow diet increased 3 fold at 20 weeks of age and then decreased to the basal level by 40 weeks of age. For oxLDL/ $\beta 2$ GPI complexes, we did not observe such a transient increase.
Natural autoantibodies are usually defined as antibodies that are formed in normal individuals in the complete absence of any exogenous antigenic stimulation. They have an important role in providing a first line of defense against invading pathogens and as such represent a nonredundant component of the humoral immune system. Natural antibodies are predominantly IgM [39]. ApoE-deficient mice fed cholesterol have very high autoantibody titers, particularly $\operatorname{IgM}$, to a wide variety of oxidation-specific epitopes [16]. The monoclonal autoantibodies secreted by hybridomas cloned from ApoE-deficient spleens were all IgM and localized by immunostaining of atherosclerotic lesions of mice and humans [19]. In our study, LDLR-deficient mice fed high fat diet showed significantly high titer of IgM antioxLDL antibodies compared with mice fed normal chow diet. PP supplementation reduced plasma cholesterol and oxLDL/32GPI complexes which contain the target of these antibodies, however, it did not significantly reduce antioxLDL antibodies.

The effect of persimmon peel on the production of natural antibody should be further investigated. However, we have noticed from a previous line of our studies that $\beta 2 \mathrm{GPI}$ is complexed with LDL once LDL is oxidized at arterial vessels and that the oxLDL/ $\beta 2$ GPI complexes are proportionally leaking out to the circulation. So, size/severity of atherosclerotic plaques can be roughly predicted by measuring plasma oxLDL/ $/ 2$ GPI complexes. Our data in the present study suggested that persimmon peel intake most probably prevents development of atherosclerosis. Beside, we also expect that minimal requirement of oxLDL present in atherosclerotic plaques to induce IgM natural autoantibodies must be relatively so small amount and that enough amount of antigenicity remains in mice fed with the HF-PP diet.

8-OHdG is one of the most abundant oxidative DNA adducts and it has been used as an indicator of oxidative 
DNA damage associated with aging. Lee et al. [4] reported that pretreatment with persimmon peel proanthocyanidin showed protective effect against oxidative damage under $\mathrm{H}_{2} \mathrm{O}_{2}$-induced cellular senescence in human fibroblasts. In this study, we didn't observe significant change in urinary 8OHdG level by persimmon peel feeding.

Adiponectin is an anti-inflammatory cytokine that is specifically and abundantly produced by adipocytes as a secretory protein and plays a key role in metabolic syndrome. The serum adiponectin concentration after 12 weeks of HF + PP feeding did not change significantly when compared with HF feeding. There was a report that ApoE-deficient mice fed high fat diet had a marked atherosclerotic lesion formation, but did not show significant difference in adipocytokine levels including adiponectin [40].

We also monitored urinary 11-dhTXB 2 during this study to clarify the effect of persimmon peel on the platelet activation. Thromboxiane $\mathrm{A}_{2}\left(\mathrm{TXA}_{2}\right)$ is the major cyclooxygenase product of arachidonic acid in platelets and a potent platelet aggregator. The biosynthesis of $\mathrm{TXA}_{2}$ increases in diseases associated with platelet activation. Since $\mathrm{TXA}_{2}$ is a very labile compound, its hydrolysis product, $\mathrm{TXB}_{2}$ has been assayed as a quantitative index of platelet activation. 11$\mathrm{dhTXB}_{2}$ was identified as the most abundant enzymatic metabolite of infused $\mathrm{TXB}_{2}$ [41] and is now considered to be the most appropriate parameter to follow the endogenous synthesis of $\mathrm{TXA}_{2}$ [42]. Aspirin is a potent inhibitor of cyclooxygenase. In LDLR-deficient mice administered lowdose aspirin, significant decrease of vascular inflammation accompanied with the decrease of urinary 2, 3-dinor- $\mathrm{TXB}_{2}$ was reported [31].

Diets rich in cholesterol and chlolate such as Paigen diet have been used to study atherogenesis and proinflammatory changes in microvasculature [43]. This diet has been shown to induce cholesterol gallstone disease in inbred mouse strains and chronic hepatic inflammation and fibrosis in rats [44], increase production of reactive oxygen species [45], and increase platelet-leukocyte interaction [46]. It has been reported that urinary $11-\mathrm{dhTXB}_{2}$ significantly increased in the cirrhotic patients in comparison with the controls [47]. In this study, LDLR-deficient mice fed HF showed modest elevation of urinary $11-\mathrm{dhTXB}_{2}$, and that was decreased by persimmon peel supplementation. There are several reports that plant polyphenol, such as green tea catechins and olive oil polyphenol, showed antiplatelet effect due to the inhibition of $\mathrm{TXA}_{2}$ formation $[48,49]$. The precise mechanism of antiplatelet activity by PP remains to be elucidated.

In conclusion, our study demonstrated that persimmon peel prevented the increment of blood cholesterol, triglyceride, and oxLDL/ $/ 32$ GPI atherogenic autoantigen levels, and prevented the progression of atherosclerosis in the LDLRdeficient mouse, but did not influence natural antibody induction against oxidized LDL. Though the precise mechanism and functional constituents of preventing atherosclerosis are not identified, persimmon peel would be beneficial in the development of preventive food supplement against dyslipidemia and atherosclerosis.

\section{ACKNOWLEDGEMENTS}

We thank to Masahiro Fujii for breeding our mouse. This study was supported in part by a Grant-in Aid for Scientific
Research from the Ministry of Education, Science, Culture and Sports of Japan.

\section{REFERENCES}

[1] Mallavadhani UV, Panda AK, Rao YR. Pharmacology and chemotaxonomy of Diospyros. Phytochemistry 1998;49:901-51.

[2] Gorinstein S, Zachwieja Z, Folta M, et al. Comparative contents of dietary fiber, total phenolics, and minerals in persimmons and apples. J Agric Food Chem 2001;49:952-7.

[3] Yokozawa T, Kim YA, Kim HY, et al. Protective effect of persimmon peel polyphenol against high glucose-induced oxidative stress in LLC-PK(1) cells. Food Chem Toxicol 2007;45:1979-87.

[4] Lee YA, Cho EJ, Yokozawa T. Protective effect of persimmon (Diospyros kaki) peel proanthocyanidin against oxidative damage under $\mathrm{H}_{2} \mathrm{O}_{2}$-induced cellular senescence. Biol Pharm Bull 2008;31:1265-9.

[5] Katsube T, Tabata H, Ohta Y, et al. Screening for antioxidant activity in edible plant products: comparison of low-density lipoprotein oxidation assay, DPPH radical scavenging assay, and FolinCiocalteu assay. J Agric Food Chem 2004;52:2391-6.

[6] Fukai S, Tanimoto S, Maeda A, et al. Pharmacological activity of compounds extracted from persimmon peel (Diospyros kaki THUNB.). J Oleo Sci 2009;58:213-9.

[7] Matsumoto K, Watanabe Y, Ohya MA, et al. Young persimmon fruits prevent the rise in plasma lipids in a diet-induced murine obesity model. Biol Pharm Bull 2006;29:2532-5.

[8] Matsumoto K, Yokoyama S, Gato N. Hypolipidemic effect of young persimmon fruit in C57BL/6.KOR-ApoEshl mice. Biosci Biotechnol Biochem 2008;72:2651-9.

[9] Steinberg D, Parthasarathy S, Carew TE, et al. Beyond cholesterol. Modifications of low-density lipoprotein that increase its atherogenicity. N Engl J Med 1989;320:915-24.

[10] Miller YI, Chang MK, Binder CJ, et al. Oxidized low density lipoprotein and innate immune receptors. Curr Opin Lipidol 2003; 14:437-45.

[11] Itabe $\mathrm{H}$, Takeshima $\mathrm{E}$, Iwasaki $\mathrm{H}$, et al. A monoclonal antibody against oxidized lipoprotein recognizes foam cells in atherosclerotic lesions. Complex formation of oxidized phosphatidylcholines and polypeptides. J Biol Chem 1994;269:15274-9.

[12] Nishi K, Itabe $\mathrm{H}$, Uno $\mathrm{M}$, et al. Oxidized LDL in carotid plaques and plasma associates with plaque instability. Arterioscler Thromb Vasc Biol 2002;22:1649-54.

[13] Itabe H, Yamamoto H, Imanaka T, et al. Sensitive detection of oxidatively modified low density lipoprotein using a monoclonal antibody. J Lipid Res 1996;37:45-53.

[14] Holvoet P, Donck J, Landeloos M, et al. Correlation between oxidized low density lipoproteins and von Willebrand factor in chronic renal failure. Thromb Haemost 1996;76:663-9.

[15] Tsimikas S, Bergmark C, Beyer RW, et al. Temporal increases in plasma markers of oxidized low-density lipoprotein strongly reflect the presence of acute coronary syndromes. J Am Coll Cardiol 2003;41:360-70.

[16] Palinski W, Ord VA, Plump AS, et al. ApoE-deficient mice are a model of lipoprotein oxidation in atherogenesis. Demonstration of oxidation-specific epitopes in lesions and high titers of autoantibodies to malondialdehyde-lysine in serum. Arterioscler Thromb 1994;14:605-16.

[17] Palinski W, Tangirala RK, Miller E, et al. Increased autoantibody titers against epitopes of oxidized LDL in LDL receptor-deficient mice with increased atherosclerosis. Arterioscler Thromb Vasc Biol 1995;15:1569-76.

[18] Palinski W, Horkko S, Miller E, et al. Cloning of monoclonal autoantibodies to epitopes of oxidized lipoproteins from apolipoprotein E-deficient mice. Demonstration of epitopes of oxidized low density lipoprotein in human plasma. J Clin Invest 1996;98:800-14.

[19] Binder CJ, Horkko S, Dewan A, et al. Pneumococcal vaccination decreases atherosclerotic lesion formation: molecular mimicry between Streptococcus pneumoniae and oxidized LDL. Nat Med 2003;9:736-43.

[20] Matsuura E, Igarashi Y, Fujimoto M, et al. Anticardiolipin cofactor(s) and differential diagnosis of autoimmune disease. Lancet 1990;336:177-8. 
[21] Matsuura E, Igarashi Y, Fujimoto M, et al. Heterogeneity of anticardiolipin antibodies defined by the anticardiolipin cofactor. J Immunol 1992;148:3885-91.

[22] Matsuura E, Igarashi Y, Yasuda T, et al. Anticardiolipin antibodies recognize $\beta 2$-glycoprotein I structure altered by interacting with an oxygen modified solid phase surface. J Exp Med 1994;179:457-62.

[23] Hasunuma Y, Matsuura E, Makita Z, et al. Involvement of B2glycoprotein I and anticardiolipin antibodies in oxidatively modified low-density lipoprotein uptake by macrophages. Clin Exp Immunol 1997;107:569-73.

[24] Kobayashi K, Matsuura E, Liu Q, et al. A specific ligand for 32 glycoprotein I mediates autoantibody-dependent uptake of oxidized low density lipoprotein by macrophages. J Lipid Res 2001;42:697709 .

[25] Kobayashi K, Kishi M, Atsumi T, et al. Circulating oxidized LDL forms complexes with $\beta 2$-glycoprotein I: implication as an atherogenic autoantigen. J Lipid Res 2003;44:716-26.

[26] Lopez LR, Hurley BL, Simpson DF, et al. Oxidized low-density lipoprotein/ $\beta 2$-glycoprotein I complexes and autoantibodies in patients with type 2 diabetes mellitus. Ann N Y Acad Sci 2005;1051:97-103.

[27] Kasahara J, Kobayashi K, Maeshima Y, et al. Clinical significance of serum oxidized low-density lipoprotein/ $\beta 2$-glycoprotein I complexes in patients with chronic renal diseases. Nephron Clin Pract 2004;98:c15-24

[28] Matsuura E, Kobayashi K, Tabuchi M, et al. Oxidative modification of low-density lipoprotein and immune regulation of atherosclerosis. Prog Lipid Res 2006;45:466-86.

[29] Kuwana M, Matsuura E, Kobayashi K, et al. Binding of $\beta 2$ glycoprotein I to anionic phospholipids facilitates processing and presentation of a cryptic epitope that activates pathogenic autoreactive T cells. Blood 2005;105:1552-7.

[30] Yamaguchi Y, Seta N, Kaburaki J, et al. Excessive exposure to anionic surfaces maintains autoantibody response to $\beta 2$ glycoprotein I in patients with antiphospholipid syndrome. Blood 2007; $110: 4312-8$

[31] Ishibashi S, Herz J, Maeda N, et al. The two-receptor model of lipoprotein clearance: tests of the hypothesis in "knockout" mice lacking the low density lipoprotein receptor, apolipoprotein E, or both proteins. Proc Natl Acad Sci USA 1994;91:4431-5.

[32] Merat S, Fruebis J, Sutphin M, et al. Effect of aging on aortic expression of the vascular cell adhesion molecule-1 and atherosclerosis in murine models of atherosclerosis. J Gerontol A Biol Sci Med Sci 2000;55:B85-94

[33] Cyrus T, Sung S, Zhao L, et al. Effect of low-dose aspirin on vascular inflammation, plaque stability, and atherogenesis in lowdensity lipoprotein receptor-deficient mice. Circulation 2002;106:1282-7.

[34] Tangirala RK, Rubin EM, Palinski W. Quantitation of atherosclerosis in murine models: correlation between lesions in the aortic origin and in the entire aorta, and differences in the extent of lesions between sexes in LDL receptor-deficient and apolipoprotein E-deficient mice. J Lipid Res 1995;36:2320-8.

[35] Hashimoto Y, Kawamura M, Ichikawa K, et al. Anticardiolipin antibodies in NZW x BXSB F1 mice. A model of antiphospholipid syndrome. J Immunol 1992;149:1063-8

[36] Gorinstein S, Bartnikowska E, Kulasek G, et al. Dietary persimmon improves lipid metabolism in rats fed diets containing cholesterol. J Nutr 1998;128:2023-7.

[37] Matsumoto K, Yokoyama S, Gato N. Bile acid-binding activity of young persimmon (Diospyros kaki) fruit and its hypolipidemic effect in mice. Phytother Res 2010;24:205-10.

[38] Kato R, Mori C, Kitazato K, et al. Transient increase in plasma oxidized LDL during the progression of atherosclerosis in apolipoprotein E knockout mice. Arterioscler Thromb Vasc Biol 2009;29:33-9.

[39] Binder CJ, Shaw PX, Chang MK, et al. The role of natural antibodies in atherogenesis. J Lipid Res 2005;46:1353-63.

[40] King VL, Hatch NW, Chan HW, et al. A murine model of obesity with accelerated atherosclerosis. Obesity (Silver Spring) 2010;18:35-41.

[41] Lawson JA, Patrono C, Ciabattoni G, et al. Long-lived enzymatic metabolites of thromboxane B2 in the human circulation. Anal Biochem 1986;155:198-205

[42] Uyama O, Shimizu S, Nakanishi T, et al. Urinary 11-dehydrothromboxane B2: a quantitative index of platelet activation in cerebral infarction. Intern Med 1992;31:735-9.

[43] Getz GS, Reardon CA. Diet and murine atherosclerosis. Arterioscler Thromb Vasc Biol 2006;26:242-9.

[44] Jeong WI, Jeong DH, Do SH, et al. Mild hepatic fibrosis in cholesterol and sodium cholate diet-fed rats. J Vet Med Sci 2005;67:235 42.

[45] Stokes KY, Cooper D, Tailor A, et al. Hypercholesterolemia promotes inflammation and microvascular dysfunction: role of nitric oxide and superoxide. Free Radic Biol Med 2002;33:1026-36.

[46] Stokes KY, Calahan L, Russell JM, et al. Role of platelets in hypercholesterolemia-induced leukocyte recruitment and arteriolar dysfunction. Microcirculation 2006;13:377-88.

[47] Davi G, Ferro D, Basili S, et al. Increased thromboxane metabolites excretion in liver cirrhosis. Thromb Haemost 1998;79:747-51.

[48] Son DJ, Cho MR, Jin YR, et al. Antiplatelet effect of green tea catechins: a possible mechanism through arachidonic acid pathway. Prostaglandins Leukot Essent Fatty Acids 2004;71:25-31.

[49] Correa JA, Lopez-Villodres JA, Asensi R, et al. Virgin olive oil polyphenol hydroxytyrosol acetate inhibits in vitro platelet aggregation in human whole blood: comparison with hydroxytyrosol and acetylsalicylic acid. Br J Nutr 2009;101:1157-64. 